\title{
Review of Premetastasis Niche Research Progress
}

\author{
Kabulo Katshi Cedric, Tingjiao Liu*, Jing Kong \\ Department of Oral Pathology, College of Stomatology, Dalian Medical University, Dalian, China \\ Email: *tingjiaoo@dlmedu.edu.cn
}

How to cite this paper: Cedric, K.K., Liu, T.J. and Kong, J. (2020) Review of Premetastasis Niche Research Progress. Journal of Biosciences and Medicines, 8, 153-162. https://doi.org/10.4236/jbm.2020.85015

Received: April 2, 2020

Accepted: May 17, 2020

Published: May 20, 2020

Copyright $\odot 2020$ by author(s) and Scientific Research Publishing Inc. This work is licensed under the Creative Commons Attribution International License (CC BY 4.0).

http://creativecommons.org/licenses/by/4.0/

\begin{abstract}
In recent years, the field of premetastasis niche has attracted more and more attention from international scholars and has become a hot research direction. This article summarizes the current research on the role of the premetastasis niche, the components and formation mechanisms of the premetastasis niche, and the research on the significance of the premetastasis niche to the clinic. Effective cancer diagnosis and treatment strategies are important. A series of research advances in this field are of great significance for further elucidating the mechanism of tumor metastasis and designing more effective cancer diagnosis and treatment strategies.
\end{abstract}

\section{Keywords}

Premetastasis Niche, Metastasis Microenvironment, Current Research, Formation Mechanisms

\section{Introduction}

The tumor microenvironment is a complex and comprehensive system, which is composed of many stromal cells, including fibroblasts, immune and inflammatory cells, adipocytes, glial cells, smooth muscle cells, and some vascular cells. The most common immune cells in the tumor microenvironment are tumor-associated macrophages (TAM) [1].

The tumor creates a microenvironment suitable for metastasis in the distant organ, that is the premetastasis niche [2]. Metastatic microenvironment is a tumor produced by the complex interaction of tumor-derived factors, tumor-mobilized bone marrow-derived cells, and host primary stromal cells. This article summarizes the current research on the role of the premetastasis niche, the components, the formation mechanisms of the premetastasis niche, and the significance of the 
microenvironment to the clinic. From this study, we can know enhanced permeability, lymphangiogenesis, organ ophilicity, and reprogramming, which will make you understand the mechanisms that promote tumor metastasis. The in-depth understanding of the microenvironmental effects, characteristics, and formation mechanisms of tumor metastasis through this study has guiding significance for the future treatment of metastatic cancer.

\section{Research on the Function of Premetastasis Niche}

Metastasis refers to the migration of tumor cells to an area that is not directly adjacent to the primary tumor. Tumor cells can spread through blood and lymphatic vessels, or penetrate directly into the spaces and spread around organs. Metastasis is a multi-stage process that requires tumor cells to shed from the primary foci, survive in circulation and grow in distant locations. Every stage of this process is affected by the tumor cells themselves and the tumor microenvironment. Some people believe that metastasis is a late-onset event in tumor development and is related to tumor volume [3]. More and more research evidences showed that the primary tumor can induce the formation of the second organ supportive microenvironment (pre-metastatic microenvironment), thereby promoting tumor metastasis [4] [5] [6]. The primary tumor will create a favorable microenvironment for the subsequent tumor metastasis in the distant organ or tissue, that is the pre-metastatic microenvironment. The formation of the premetastasis niche requires the interaction of tumor-derived factors, bone marrow-derived cells mobilized by the tumor, and local mesenchymal cells.

Proteases in the microenvironment provide a material basis for tumor metastasis. Tumor metastasis is accompanied by a variety of proteolytic activation, and this process is performed by a series of protein kinases, which is widely accepted. The matrix metalloproteinase (MMP) family is currently the most widely studied and considered to be the most closely related protease in tumors, because it is often up-regulated in tumors and can degrade ECM after secretion or localization on the cell surface, thus destroy the histological barrier of tumor cell invasion [3]. Hematopoietic stem cells overexpressing MMP9 induce malignant transformation of normal tissues and establish a pre-metastatic microenvironment, pre-metastatic niche plays an important role. The expression and secretion of MMP9 are regulated by VEGF and the chemokine ligand CXCL12/receptor CXCR4 axis, and the last two of them are also important factors that constitute the pre-metastatic microenvironment [7]. At the same time, CAF can secrete chemokine ligands (CXCL1, CXCL2, CXCL5) and MMPs (MMP3, MMP12), etc., while TAM can secrete MMP1, MMP2, MMP7, MMP9, MMP12, MMP14, a large part of which are related to tumors, the occurrence, development and neovascularization are closely related [8]. In addition, studies have shown that most MMPs produced by stromal cells are generally elevated in tumor cells and are associated with tumor infiltration and metastasis. Because MMPs can decompose 
and reshape ECM and basement membrane (BM), they can promote tumor metastasis. MMPs are also related to the cleavage of a large number of cell surface molecules and soluble proteins, such as epithelial concept proteins, integrins, $\mathrm{T}$ cells (CD44), interleukin-8 (IL-8), and membrane cofactor proteins (membrancecoprotein (MCP-3), chemokine stromal cell-derived factor (stromal-derived factor-1, SDF-1), etc. [7]. Recently, other proteases that make up the tumor microenvironment have gradually received more attention. For example, the cathepsin family, which functions similarly to MMPs, and some members are often up-regulated during tumorigenesis. Once localized on the cell surface, can also break down ECM and promote tumor metastasis [9].

Cathepsin is generally stable in the acidic environment of lysosomes and is capable of breaking down a range of substrates. In addition, there is evidence that cathepsin can also play a role in cells (such as cytosol, nucleus), cell surface and extracellular cells [10]. Both intracellular and extracellular cathepsin can cleave the major components of ECM and basement membrane, such as Laminin, Fibronectin and Collagentype IV, thereby promoting tumor cell infiltration and metastasis [11]. Although most cathepsin is constitutively expressed and functions in the lysosome, some cathepsin are overexpressed in tumor cells (such as cathepsin $\mathrm{H}$, cathepsin L), and some are induced in non-tumor cells that constitute the tumor microenvironment. Some studies have investigated the potential application value of microenvironment before tumor metastasis and re-tumor prevention and treatment, the research mainly discussed in two aspects: 1) By blocking the generation of molecules that are beneficial to the formation of microenvironment before tumor metastasis; 2) Finding biomarkers that can predict tumor metastasis [12] (as shown in Figure 1).
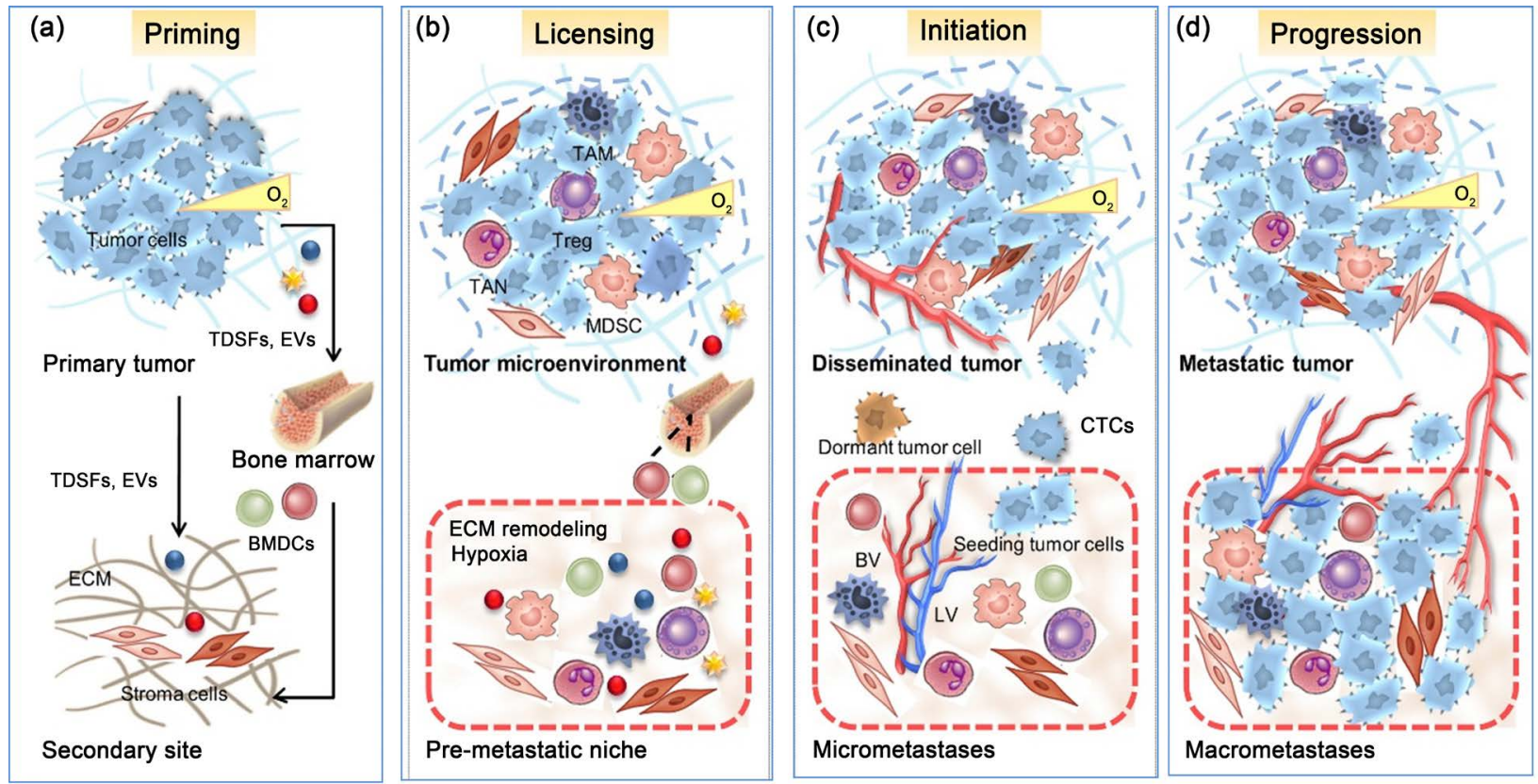

Figure 1. Role of the pre-metastatic niche in the promotion of tumor [12]. 


\section{Research on the Formation Mechanism of Premetastasis Niche}

This paper describes the molecular and cellular composition of the microenvironment before tumor metastasis and its regulatory mechanisms. In situ tumors can induce the formation of a microenvironment that is conducive to tumor cell metastasis in specific organs and tissues before metastasis. The formation of the microenvironment before tumor metastasis requires tumor secretion factors, mobilization of suppressive immune cells, and matrix components of the tissue site. The interaction of three major factors, including inflammatory polarization. In addition, local hypoxia and extracellular matrix remodeling also promote the formation of a microenvironment before tumor metastasis. At the same time, the article comprehensively summarizes the key molecules and cellular components that promote the formation of the microenvironment before tumor metastasis and the underlying mechanisms, and first proposes six characteristics of the microenvironment before tumor metastasis: immunosuppression, inflammatory response, angiogenesis and permeability. Enhancement, lymphangiogenesis, pro-organism, and reprogramming provide new insights into the mechanism of the pre-metastatic microenvironment to promote tumor metastasis [12]. Each of the six features of the tumor process, including tumor metastasis, can be cleared by the immune system. CD8 + T cells, natural killer cells (NK), and atypical monocytes can prevent tumor metastasis without affecting the growth of the primary tumor [13]. Bone marrow-derived suppressor cells that accumulate in the premetastasis niche can also pass ARG1 and production of reactive oxygen species (ROS) inhibits anti-tumor T cells [14].

TNF receptor-2 can support bone marrow-derived suppressor cell-mediated immunosuppressive processes, promote tumor cell planting, growth and metastasis in the formation of a microenvironment before liver metastasis [15]. In lung, bone marrow, a large increase in immuno suppressive and metastasis-promoting T-regulatory cells has been demonstrated in a variety of cancers [16]. Metastatic cells in the tissue such as alveolar macrophages pretreated by breast cancer cells, can establish an immunosuppressive microenvironment by suppressing anti-tumor T cells [17]. Chronic inflammation is an important factor in tumor development and metastasis. There are many molecular components and signaling pathways involved in regulating immune cells, inflammation, tumor growth and metastasis. For example, abnormal activation of TLR4 signaling in tumor cells can lead to inflammatory responses, tumor cell death resistance, and increase their proliferation and invasion capabilities. Similarly, abnormal activation of TLR4 and other innate immune cell sensing pathways leads to an inflammatory response that promotes tumor progression and metastasis [18]. TNF- $\alpha$-induced S100A8SAA3-TLR4 signaling pathway can also stimulate clara cells, thereby expressing SAA3 and maintaining the inflammatory state of the microenvironment before lung metastasis, to promote the occurrence of tumor lung met astasis [19]. $\mathrm{CD} 11 \mathrm{~b}+\mathrm{Gr}-1+$ cells in the microenvironment before lung metastasis can also 
express MMPs and TH2 cells factor, reducing the production of IFN- $\gamma$, transforming the microenvironment into an inflammatory and proliferative microenvironment [19]. Upregulation of IL-6 by TLR5 and the hypoxic environment in the pre-metastatic microenvironment can drive tumor-promoted inflammatory responses. Therefore, before circulating tumor cells reach the site of metastasis, an inflammatory environment is established in the secondary site [20], which is conducive to the planting, survival, and proliferation of tumor cells in the premetastasis niche.

Enhanced angiogenesis and permeability, the microenvironment prior to metastasis may increase angiogenesis and vascular permeability, thereby promoting metastasis. TIE $2+$ monocytes and endothelial progenitor cells recruited in the pre-metastatic microenvironment to create a pro-stimulatory agent with high concentrations of VEGF and other pro-angiogenic factors.

The microenvironment of angiogenesis can initiate angiogenesis and promote the metastatic process in tumor progression [20]. In the pre-metastatic microenvironment, calcium-dependent phosphatase and activated $\mathrm{T}$ cell nuclear factor (NFAT) pathways can be specifically activated by lung endothelium before tumor cells arrive, due to NFAT-targeted angiogenin-2 (ANG-2), Leading to an increase in lung metastasis [21]. In the early formation of the premetastasis niche, miR105 secreted by metastatic breast cancer cells can damage the vascular endothelial barrier through tight junction of targeted cell tight junction proteins, thereby enhancing vascular permeability of distant organs and promoting metastasis [22]. In addition, the epidermal growth factor receptor ligand epidermal regulator, cyclooxygenase-2, MMP-1 and MMP-2 expressed by breast cancer cells recombine a multifunctional vascular remodeling program to increase vascular permeability and promote Pulmonary metastasis process [23].

Lymphangiogenesis in the premetastasis niche is an active step in tumor metastasis, and lymphatic vessels may serve as a starting site for lymphatic dissemination of tumors. Clinical data show that tumor-derived VEGF-A and VEGF-D generate lymphatic vessels before lymph node-induced metastasis, and are associated with lymph node metastasis [24]. The mechanism of lymphatic metastasis, especially the role of lymphatics in metastasis in the premetastasis niche is not clear. In addition, the permeability of the lymphatic vessels and low lymphatic flow further illustrate the importance of lymphangiogenesis in tumor metastasis. Further analysis of the secreted proteins of LECs and the identification of key components of lymphangiogenesis in metastasis can help us to explore new targets for inhibiting tumor metastasis. The organophilic feature is congenitally related to the pre-metastatic microenvironment, that is certain types of cancer can easily transfer to specific organs through a selective microenvironment. Tumors select specific organs for metastasis by secreting tumor-derived secretion factors to change the adhesion molecules and extracellular matrix at these sites. Certain factors can also promote metastasis by mobilizing bone marrow-derived cells to specific secondary organs and establishing a microenvironment that facilitates 
metastasis. Therefore, the increase in molecular and cellular components selectively in different pre-metastatic microenvironments makes tumor metastases more organophilic. Reprogramming involves metastatic reprogramming, matrix reprogramming, and gene reprogramming in tumor metastasis promoted by the premetastasis niche. Differences in metabolic reprogramming determine where tumor cells metastasize to organs. For example, thrombopoietin promotes metastasis of colon tumor-initiating cells to the liver by increasing lysine metabolism in tumor-initiating cells and promotes liver transplantation. This explains why colon cancer preferentially and selectively metastasizes to the liver [25]. Tumor cells may recruit and reprogram benign stromal cells to the pre-metastatic microenvironment to promote tumor growth and metastatic spread. The six characteristics of the premetastasis niche make it capable of promoting tumor metastasis. In addition, the pre-metastatic microenvironment also promotes metastasis in other ways, such as providing survival signals to circulating tumor cells. For example, VCAM-1 expressing tumor cells require a survival signal from macrophages before they metastasize in the lungs, and metastasize to the lungs through direct intracellular action. In summary, Immunosuppression, inflammation, angiogenesis/vascular permeability, lymphangiogenesis, organophilic and reprogramming may mediate function through different but integrated approaches, thereby promoting tumor metastasis (as shown in Figure 2).

\section{Research on the Clinical Significance of Premetastasis Niche}

Studies have shown that in a mouse model of breast cancer, the spread of tumor cells has nothing to do with tumor size, and it has occurred before tumor invasion.

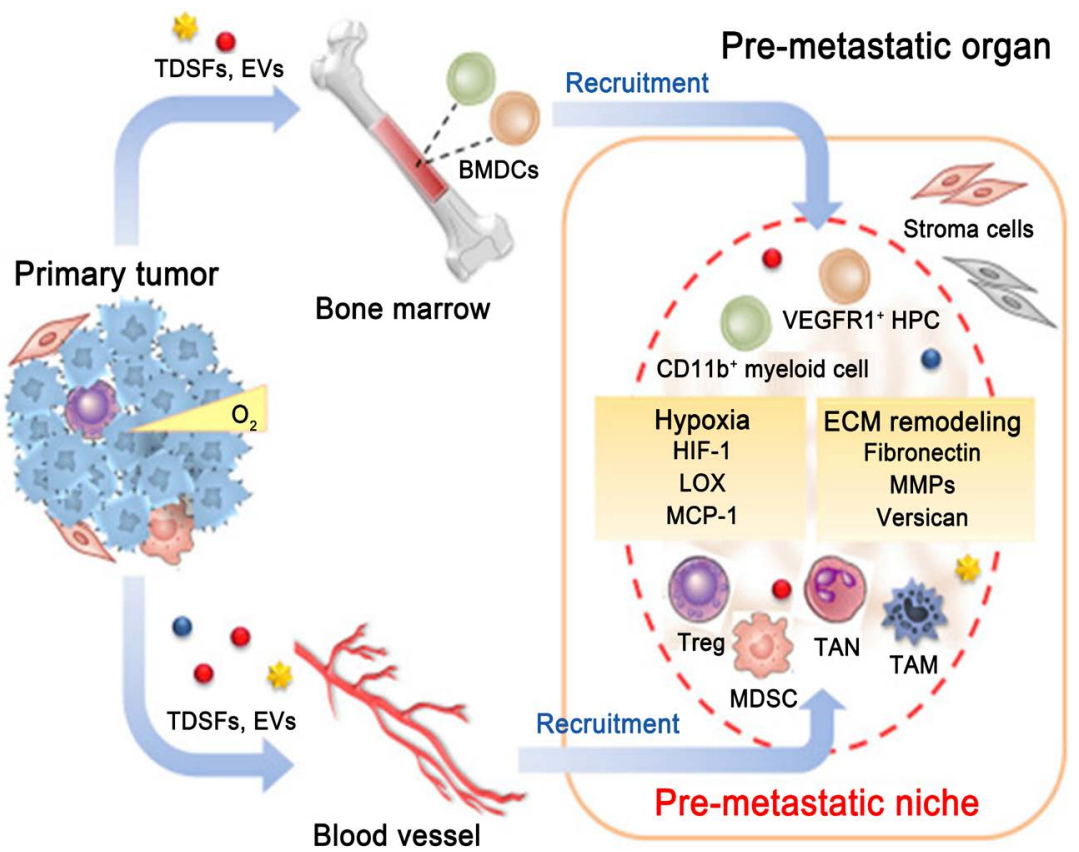

Figure 2. Induction and formation of the pre-metastatic niche [11]. 
In addition, even non-malignant positive mammary epidermal cells can survive in the lungs for a long time, and will develop into (lung) metastases after rapid or slow oncogene activation, which suggests that cell proliferation and planting in other parts can be happened before the malignant change [26]. Recently, some scholars believe that the primary tumor is nourished by circulating tumor cells. According to this "self-implantation", even in the early stages of tumorigenesis, a large number of tumor cells have appeared in the circulation, and were found far earlier than distant metastases [26]. Some research results have found that the regulation of the microenvironment before liver metastasis, such as the activation of various cells, the regulation of immune responses, and changes in cytokine levels, play a decisive role in the occurrence and development of liver metastases in digestive system tumors [27].

Animal experiments have confirmed that a large number of inflammatory factors have accumulated in the lungs before the formation of lung metastases. Changes in host target organs affect every step of metastasis formation. Bethan Psaila and David Lyden proposed the pre-metastatic niche and metastatic niche hypotheses for this soil change process, pointing out that soil changes are the guarantee of metastasis formation. The hypothesis quickly caused a sensation in the tumor research community. Studying this series of changes in cell and molecular biology will help clarify the complex process of tumor metastasis and bring new ideas for metastatic intervention [28]. By screening the model of spontaneous lung metastasis of tumors in situ established by innate immune receptor-deficient mice, it was found that TLR3 innate immune receptor-deficient mice had significantly reduced tumor lung metastasis and prolonged survival of tumor-bearing mice $(\mathrm{p}<0.05)$. In-depth research shows that the natural immune receptor TLR3 expressed by type II lung epithelial cells recruits neutrophils to accumulate in the lungs by recognizing exosomal RNA secreted by the tumor into the blood circulation and triggering inflammatory reactions such as chemokine release. An inflammatory microenvironment was formed locally in the lungs, creating conditions for tumor lung metastasis. This discovery provides new intervention targets and ideas for tumor treatment, especially tumor metastasis prevention and treatment [29]. The continued survival of tumor cells is the main rate-limiting link in the process of tumor metastasis. Similar to the tumor microenvironment, the local microenvironment of the organ before metastasis (premetastasis niche) provides a suitable environment for the survival of tumor cells and is an important condition for the continued survival and proliferation of tumor cells in remote organs. Myeloid-derived suppressor cells (MDSCs) are the key components in this environment, and their pre-metastatic microenvironment in tumor cell colonization and metastasis is promoted by proliferation, inflammation, immunosuppression, and vascular remodeling. Foci play an important role in the formation of foci and are potential targets for anti-tumor metastatic therapy. MDSCs are activated by signal pathways such as S1PR1-STAT3 and TGF $\beta$ under the action of various factors secreted by tumor cells and are re- 
cruited into the pre-metastatic microenvironment. They secrete a variety of proproliferative, pro-inflammatory, and immunosuppressive factors and make local blood vessels highly permeable. Constructing a premetastasis niche that can promote the recruitment, planting and expansion of tumor cells, and provide conditions for the formation of metastases. Intervening the accumulation of MDSCs in the pre-metastatic microenvironment can inhibit early tumor metastasis, which is an innovation in tumor treatment concepts and methods, and has great significance for the long-term survival of tumor patients [30].

\section{Conclusion}

A better understanding of the premetastasis niche formation mechanisms, identification of the function and characteristics, and their effect on tumor metastasis will provide invaluable insights and new strategies for preventing and treating metastatic cancer. The tumor microenvironment affects the biological characteristics of tumor cells through the action of specific factors. In-depth research on the signaling and molecular mechanisms of the interaction between tumor cells and the microenvironment will provide us with more information to study the reversion of malignant phenotypes of tumor cells and the maintenance of normal gene phenotypes for the diagnosis and treatment of tumors that provide new ways.

\section{Conflicts of Interest}

The authors declare no conflicts of interest regarding the publication of this paper.

\section{References}

[1] Mantovania, S. (2010) Macrophages, Innate Immunity and Cancer: Balance, Tolerance, and Diversity. Current Opinion in Immunology, 22, 231-237. https://doi.org/10.1016/j.coi.2010.01.009

[2] Peinado, H., Zhang, H., Matei, I., et al. (2017) Pre-Metastatic Niches: Organ-Specific Homes for Metastases. Nature Reviews Cancer, 17, 302-317. https://doi.org/10.1038/nrc.2017.6

[3] Kaplan, R.N., Riba, R.D., Zacharoulis, S., et al. (2005) VEGFR1-Positive Haematopoietic Bone Marrow Progenitors Initiate the Pre-Metastaticniche. Nature, 438, 820-827. https://doi.org/10.1038/nature04186

[4] Sleeman, J.P. (2012) The Metastatic Niche and Stromal Progression. Cancer Metastasis, 31, 429-440. https://doi.org/10.1007/s10555-012-9373-9

[5] Chin, A.R. and Wang, S.E. (2016) Cancer Tills the Premetastatic Feld: Mechanistic Basis and Clinical Implications. Clinical Cancer, 22, 3725-3733. https://doi.org/10.1158/1078-0432.CCR-16-0028

[6] Hanahan, D. and Weinberg, R.A. (2000) The Hall Marks of Cancer. Cell, 200, 57-70. https://doi.org/10.1016/S0092-8674(00)81683-9

[7] Noel, A., Jost, M. and Maquoi, E. (2008) Matrix Metalloproteinases at Cancer Tumor-Host Interface. Seminars in Cell and Developmental Biology, 19, 52-60. https://doi.org/10.1016/j.semcdb.2007.05.011 
[8] Kessenbrock, K., Plaks, V. and Werb, Z. (2010) Matrix Metalloproteinases: Regulators of the Tumor Microenvironment. Cell, 141, 52-67. https://doi.org/10.1016/j.cell.2010.03.015

[9] Van Damme, J., Struyf, S. and Opdenakker (2004) Chemokine-Protease Interactions Cancer. Seminars in Cancer Biology, 14, 201-208. https://doi.org/10.1016/j.semcancer.2003.10.007

[10] Mohamed, M.M. and Sloane, B.F. (2006) Cysteine Cathepsins: Multi-Functional Enzymes in Cancer. Nature Reviews Cancer, 6, 764-775. https://doi.org/10.1038/nrc1949

[11] Reiser, J., Adair, B. and Reinheckel, T. (2010) Specialized Roles for Cysteine Cathepsins in Health and Disease. Journal of Clinical Investigation, 120, 3421-3431. https://doi.org/10.1172/JCI42918

[12] Liu, Y. and Cao, X. (2016) Characteristics and Significance of the Pre-Metastatic Niche. Cancer Cell, 30, 668-681. https://doi.org/10.1016/j.ccell.2016.09.011

[13] Bidwell, B.N., Slaney, C.Y., Withana, N.P., et al. (2012) Silencing of Irf7 Pathways in Breast Cancer Cell Promotes Bone Metastasis through Immuneescape. Nature Medicine, 18, 1224-1231. https://doi.org/10.1038/nm.2830

[14] Tacke, R.S., Lee, H.C., Goh, C., et al. (2012) Myeloid Suppressor Cells Induced by Hepatitis C Virus Suppress T-Cell Responses through the Production of Reactive Oxygen Species. Hepatology, 55, 343-353. https://doi.org/10.1002/hep.24700

[15] Ham, B., Wang, N.D., Costa, Z., et al. (2015) TNF Receptor-2 Facilitates an Immunosuppressive Microenvironment in the Liver Topromote the Colonization and Growth of Hepatic Metastases. Cancer Research, 75, 5235-5247. https://doi.org/10.1158/0008-5472.CAN-14-3173

[16] Lai, C., August, S., Behar, R., et al. (2015) Characteristics of Immune Suppressive Regulatory T Cells in Cutaneous Squamous Cell Carcinomas and Role in Metastasis. The Lancet, 385, 59. https://doi.org/10.1016/S0140-6736(15)60374-9

[17] Sharma, S.K., Chintala, N.K., Vadrevu, S.K., et al. (2015) Pulmonary Alveolar Macrophages Contribute to the Premetastatic Niche by Suppressing Antitumor T Cell Responses in the Lungs. The Journal of Immunology, 194, 5529-5538. https://doi.org/10.4049/jimmunol.1403215

[18] Cao, X. (2016) Self-Regulation and Cross-Regulation of Pattern-Recognition Receptor Signal in Health and Disease. Nature Reviews Immunology, 16, 35-50. https://doi.org/10.1038/nri.2015.8

[19] Tomita, T., Sakurai, Y., Ishibashi, S. and Maru, Y. (2011) Imbalance of Clara Cell-Mediated Homeostatic Inflammation Is Involved in Lung Metastasis. Oncogene, 30, 3429-3439. https://doi.org/10.1038/onc.2011.53

[20] Yan, H.H., Pickup, M., Pang, Y., et al. (2010) Gr-1+CD11b+ Myeloid Cells Tip the Balance of Immune Protection to Tumor Promotion in the Premetastatic Lung. Cancer Research, 70, 6139-6149. https://doi.org/10.1158/0008-5472.CAN-10-0706

[21] Minami, T., Jiang, S., Schadler, K., et al. (2013) The Calcineurin-NFAT-Angiopoietin-2 Signaling Axis in Lung Endothelium Is Critical for the Establishment of Lung Metastases. Cell Reports, 4, 709-723. https://doi.org/10.1016/j.celrep.2013.07.021

[22] Zhou, W., Fong, M.Y., Min, Y., et al. (2014) Cancer-Secreted miR-105 Destroys Vascular Endothelial Barriers to Promote Metastasis. Cancer Cell, 25, 501-515. https://doi.org/10.1016/j.ccr.2014.03.007

[23] Gupta, G.P., Nguyen, D.X., Chiang, A.C., et al. (2007) Mediators of Vascular Remodeling Co-Opted for Sequential Steps in Lung Metastasis. Nature, 446, 765-770. https://doi.org/10.1038/nature05760 
[24] Wang, H., Yu, C., Gao, X., et al. (2015) The Osteogenic Niche Promotes Early-Stage Bone Colonization of Disseminated Breast Cancer Cells. Cancer Cell, 27, 193-210. https://doi.org/10.1016/j.ccell.2014.11.017

[25] Podsypanina, K., Du, Y.C., Jechlinger, M., et al. (2008) Seeding and Propagation of Untransformed Mouse Mammary Cells in the Lung. Science, 321, 1841-1844. https://doi.org/10.1126/science.1161621

[26] Kim, M.Y., Oskarsson, T., Acharyya, S., et al. (2009) Tumor Self-Seeding by Circulating Cancer Cells. Cell, 139, 1315-1326. https://doi.org/10.1016/j.cell.2009.11.025

[27] Xiao, H. and Xiao, Y.P. (2017) Role of Liver Microenvironment in Liver Metastasis of Digestive System Tumors and Its Regulatory Mechanism. Chinese Journal of Clinicians (Electronic Edition), 11, 32-35.

[28] Zheng, Y. and Long, H. (2011) Pre-Metastatic Microenvironment and Metastatic Microenvironment of Lung Metastases. Chinese Journal of Clinical Thoracic and Cardiovascular Surgery, 18, 555-560.

[29] Liu, Y., Gu, Y., Han, Y., et al. (2017) Study on the Important Function and Mechanism of Lung Epithelial TLR3 in the Formation of Microenvironment before Tumor Metastasis. Science News, No. 4, 58.

[30] Wei, H.M. (2017) The Role of Myeloid-Derived Suppressor Cells in the Construction of Microenvironment to Promote Tumor Metastasis before Metastasis. Chinese Journal of Cancer, 6, 516-520. 